\title{
Challenges for dedicated smoking cessation services in developing countries
}

\author{
G Y Tadzimirwa, ${ }^{1} \mathrm{MB}$ ChB; C Day, ${ }^{1} \mathrm{MB}$ BCh, Dip HIV Man (SA), MMed (Medicine); A Esmail, ${ }^{1}$ MD, FCP (SA), Cert Pulmonology (SA); \\ C Cooper, ${ }^{2}$ Dip Nurs; M Kamkuemah, ${ }^{3} \mathrm{MPH}$; K Dheda, ${ }^{1,4} \mathrm{MB}$ BCh, FCP (SA), FCCP, FRCP, PhD; \\ R N van Zyl-Smit, ${ }^{1,4}$ MB ChB, MRCP (UK), Dip HIV Man SA, FCP (SA) MMed, Cert Pulm (SA), PhD \\ ${ }^{1}$ Division of Pulmonology, Department of Medicine, Faculty of Health Sciences, University of Cape Town, South Africa \\ ${ }^{2}$ Division of Nursing, E16 Respiratory Clinic, Groote Schuur Hospital, Cape Town, South Africa \\ ${ }^{3}$ Division of Public Health Medicine, School of Public Health and Family Medicine, Faculty of Health Sciences, University of Cape Town, South Africa \\ ${ }^{4}$ University of Cape Town Lung Institute, South Africa
}

Corresponding author: R N van Zyl-Smit (richard.vanzyl-smit@uct.ac.za)

\begin{abstract}
Background. South Africa, ranked as the world's second most stressful country to live in, has an estimated 7 million smokers. A dedicated smoking cessation clinic established at Groote Schuur Hospital, Cape Town, provides the only clinical service and training centre in the country.

Objectives. To evaluate the smokers attending the clinic, in order to better understand the requirements of smoking cessation services in resource-limited settings.

Methods. Demographic and smoking-related data were collected prospectively from all clinic attendees since its inception. Nicotine dependence, depression scores and exhaled carbon monoxide levels were formally evaluated. Consent was provided to review the data collected.

Results. Ninety-seven smokers were evaluated. Their mean (standard deviation) age was 50.9 (10.7) years, and 59\% (57/97) were male. The median age of smoking initiation was 16 years (interquartile range (IQR) 8 - 28), with a current median daily consumption of 12 cigarettes (IQR 7 - 20). Overall, men smoked more than women, with a median of 20 cigarettes per day (IQR 10 - 20) v. 12 (IQR 5 - 20), respectively $(p=0.001)$. The median Fagerström nicotine dependence score was 5 (IQR 3 - 7), with scores of 6 (IQR 4 - 8) for men and 5 ( 2 - 7) for women ( $p=0.06$ ); $50 \%$ of smokers had a Fagerström score $<6$ (low to above-average dependence) and $22 \%$ a score $\geq 8$ (extreme dependence). The median Patient Health Questionnnaire-9 (PHQ-9) depression score was 8 (IQR 4 - 11), and 49\% of smokers had symptoms of at least minor depression (score $\geq 10$ ). The clinic could not provide pharmacotherapy. The self-reported quit rate was $28 \%$ at median follow-up of 22 months (IQR 14 - 39).

Conclusions. In smokers attempting to quit, moderate levels of nicotine dependence coexist with significant depression and anxiety symptoms. These data inform resource allocation and public health strategies, suggesting that in resource-limited smoking cessation services, psychological/behavioural support focusing on depressive symptoms may be a greater priority than simple pharmacotherapy.
\end{abstract}

S Afr Med J 2019;109(6):431-436. DOI:10.7196/SAMJ.2019.v109i6.13631

The detrimental effect of smoking on population health has been apparent since the early 1900s, when a relationship between increased prevalence of smoking and increased incidence of lung cancer, previously uncommon, was identified. ${ }^{[1,2]}$ Since then, numerous studies have highlighted the relationships between smoking and non-communicable diseases including cardiorespiratory disease, autoimmune disease, subfertility, malignancy, cerebrovascular disease and poor fetomaternal outcomes. ${ }^{[2-6]}$

South Africa (SA)'s first burden of disease report in 2000 highlighted the quadruple burden of disease the country faces and the need for a multifaceted approach to improving health that includes managing modifiable risk factors, particularly smoking. ${ }^{[7]}$ It was reported that in SA in 2000, smoking accounted for $8.5 \%$ of all deaths (and 13\% of deaths in adults aged $>35$ years) and $3.7-4.3 \%$ of disability-adjusted life-years due to lung and other cancers, cardiovascular disease, chronic obstructive pulmonary disease (COPD), tuberculosis and other medical conditions. ${ }^{[8]}$ South Africa has an estimated 7.2 million adults living with HIV, $60 \%$ of whom are co-infected with TB, with $61 \%$ of adults on antiretroviral therapy ${ }^{[9]}$ In addition, both smoking and $\mathrm{TB}$ increase the risk of COPD. ${ }^{[10-13]}$ However, individuals who quit smoking successfully lower their risk with every year after cessation. Furthermore, those who manage to quit before the age of 45 have the same life expectancy as those who have never smoked. ${ }^{[14]}$

Many low- and middle-income countries (LMICs) have made significant efforts to minimise adverse health outcomes due to smoking, with legislation restricting tobacco use, advertising and trade following the World Health Organization (WHO) MPOWER strategy. ${ }^{[15]}$ Formal cessation programmes mainly exist in high-income countries, which equate to $\sim 15 \%$ of the world's population able to access cessation support. ${ }^{[16]}$ Very few data exist on formal cessation programmes provided in low-income settings or on how applicable cessation strategies developed in high-income settings are to low-income countries. ${ }^{[16,17]}$ Furthermore, smoking cessation pharmacotherapy such as nicotine replacement therapy (NRT), although on the WHO essential medicine list, is not always available to smokers. ${ }^{[18,19]}$ This is particularly true in SA, where, although it is classed as a middleincome country, no smoking cessation pharmacotherapy is available to smokers accessing public health services.

A combination of behavioural therapy and pharmacological support is regarded as the interventional strategy with the highest 
odds of long-term cessation. There are, however, no data to indicate who will be unsuccessful if pharmacotherapy is not added to behavioural interventions.

\section{Objectives}

A dedicated smoking cessation clinic established at Groote Schuur Hospital (GSH) in Cape Town is the only one of its kind in SA. Our objectives were to to assess the sociodemographic and dependency profiles of the smokers who attended this clinic and their short- and long-term outcomes. By understanding the characteristics and needs of smokers in this setting, we hoped to provide relevant data to help guide locally applicable smoking cessation services applicable in LMICs. Identifying factors that support successful quitting, and those that act as barriers, is imperative in understanding the requirements for psychological and pharmacological support in such services.

\section{Methods \\ Establishing the clinic}

The first SA smoking cessation clinical practice guidelines were published in 2013. ${ }^{[19]}$ Prior to this date and the establishment of the GSH clinic in December 2012, no formal smoking cessation services existed in the public sector. No pharmacotherapy was available for smoking cessation in government hospitals, and availability of nicotine patches was very limited owing to available products being withdrawn from SA. With permission from the Head of the Division of Pulmonology at GSH, a specialised clinic was introduced to offer evaluation of and smoking cessation support to any smoker wishing to quit. The clinic was established within the respiratory outpatient services for convenience, as the trained staff were from the Division of Pulmonology and the clinic was physically near the cardiology outpatients and transplant services. The doctors had received limited smoking cessation training at a variety of local workshops, and a nursing sister was trained to provide additional counselling. No specialist psychological/psychiatric support or pharmacotherapy was available in the clinic.

The study reviewed data collected from all smokers attending GSH's smoking cessation clinic since its inception. New patient data collection commenced when the clinic opened in 2012 and was truncated in September 2016. A pre-formed data capturing sheet to guide the consultation and to assimilate the required smoking cessation-specific information was used. Self-reported scales (Fagerström, Patient Health Questionnnaire-9 (PHQ-9), etc.) were completed by the individual person, with the remaining evaluation completed by the attending doctor. Information captured included age, motivation to quit, tobacco smoking history, medical history, Fagerström score, Wisconsin withdrawal score, PHQ-9 depression score and perceived medication affordability. Carbon monoxide (CO) levels were tested using a piCO Smokerlyzer (Bedfont, UK) at each visit.

\section{Interventions}

After completing the prespecified questionnaires, all attendees were seen by a doctor (pulmonologist or medical registrar). Basic smoking history was confirmed, and level of motivation and reasons for wishing to quit were evaluated. Counselling was performed by the doctor, as there were limited alternative counselling personnel. Counselling included evaluation of previous attempts to quit and reasons for relapse or failure. Information on nicotine dependence was provided, as well as on the importance of behavioural change and pharmacotherapy if needed. Interviews were conducted in a motivational interviewing style directed towards behavioural change with a specific focus on self-motivation, problem solving and strategies for coping with withdrawal symptoms.

\section{Ethical considerations}

Ethics approval to perform this clinical patient database review was granted by the University of Cape Town Faculty of Health Sciences Human Research Ethics Committee (ref. no. 667/205) and GSH administration. At the time of their first visit, all patients provided written informed consent to have their data relevant to the smoking cessation clinic collected as part of the standard evaluation of all patients at the clinic. Participants also provided contact details to allow for telephonic contact to provide support and evaluate outcomes.

\section{Statistical analysis}

Data were entered onto an electronic database and independently reviewed for accuracy. Analysis was conducted using Graphpad prism 5.0 for Mac OSX (GraphPad Software, USA) and Stata 13 (StataCorp, USA). Continuous data were expressed as means (standard deviations (SDs)) or medians with interquartile ranges (IQRs). For categorical data, the $\chi^{2}$ test or Fisher's exact test was used. The Mann-Whitney test or Student's $t$-test was used to test the association between continuous and categorical variables. A $p$-value of 0.05 was used to determine statistical significance.

\section{Results}

\section{Participants}

The weekly smoking cessation clinic received the majority of its referrals from cardiology, respiratory and other tertiary clinics (organ transplant, infertility) at GSH. A few attendees were 'healthy smokers' from outside the hospital. During the audit review, 98 smokers attended for at least one visit. Only 1 attendee did not complete the requisite forms and was not included in any of the analyses.

Detailed characteristics of the patients attending the clinic are reported in Table 1 . There was a predominance of male smokers $(60 \%$, 58/97). The mean (SD) age of attendees was 51.1 (10.9) years. There was no difference in the age of men compared with women, and the median age of smoking initiation was similar at 16 years (IQR 8 - 28). On average, men smoked more cigarettes per day than women (median 20 (IQR $10-20$ ) v. 12 (IQR 5 - 20), respectively; $p=0.001$ ), resulting in higher cumulative total pack-years of smoking at the time of the first visit (33 (IQR 20 - 45) v. 21 (IQR 7 - 33); $p=0.001$ ). However, at the time of the first visit the majority of attendees were smoking 12 cigarettes per day on average. The most frequent motive noted for quitting was, not unexpectedly, health reasons (Table 2).

The majority of attendees had previously tried to quit: $91 \%$ had made at least one attempt within the past year, with $48 \%$ making more than four attempts in the past 12 months. Only $8 \%$ reported having previously used pharmacotherapy in a quit attempt. Of the attendees, $68 \%$ lived with other smokers in the house. One-third (32\%) indicated at the initial visit that they could potentially afford to purchase medication privately to assist with the quit attempt (Table 2).

Nicotine dependence was evaluated using the Fagerström Test. ${ }^{[20]}$ The median score was 6 (IQR 3 - 7). Women had slightly lower scores than men (median 5 (IQR 2 - 7) v. 6 (IQR 4 - 8), respectively; $p=0.06$ ), as shown in Table 2 . However, $50 \%$ of smokers had a score of 4 - 5 , indicating 'above-average' dependence, and $23 \%$ had a score $>8$, indicating 'extreme dependence' (Fig. 1). Exhaled CO levels were similar in men and women, with a mean (SD) score of 15.9 (9.1) parts per million. Exhaled CO correlated positively with current tobacco consumption (Spearman's $r=0.52$ ( $95 \%$ confidence interval (CI) $0.28-0.70) ; p<0.001)$ ) and with Fagerström scores, although 
Table 1. Attendee characteristics

\begin{tabular}{llll}
\hline Characteristics & Female $(\mathbf{N = 3 9 , 4 0 \% )}$ & Male $(\mathbf{N = 5 8 , 6 0 \% )}$ & Total $(\mathbf{N = 9 7 )}$ \\
\hline Current age (years), mean (SD) & $48.5(9.5)$ & $52.4(11.3)$ & $50.9(10.7)$ \\
Age at smoking initiation (years), median (IQR) & $16(15-19)$ & $16(14-18)$ & $16(14.5-18)$ \\
Total years smoked, mean (SD) & $31.7(10.1)$ & $34.7(11.9)$ & $33.5(11.3)$ \\
Average number per day smoked, median (IQR) & $12(5-20)^{*}$ & $20(10-20)^{*}$ & $20(10-20)$ \\
Current number per day smoked, median (IQR) & $11(8-17)$ & $15(6-25)$ & $12(7-20)$ \\
Total pack-years smoked, median (IQR) & $21(7-33)^{*}$ & $33(20-45)^{*}$ & $29.5(12.5-40)$ \\
Previous attempt to quit smoking, $n(\%)$ & $32(82)$ & $43(75)$ & $75(78)$ \\
Quit attempts in the past year, $n(\%)$ & & & $9(9)$ \\
$\quad$ None & $3(8)$ & $6(11)$ & $41(43)$ \\
$\quad 1-4$ & $18(46)$ & $23(40)$ & $46(48)$ \\
$\quad>4$ & $18(46)$ & $28(49)$ & $65(68)$ \\
Lives with smoker in house, $n(\%)$ & $28(72)$ & $37(65)$ & $31(32)$ \\
Number of smokers in house, $n(\%)$ & & & $38(40)$ \\
$\quad 0$ & $11(28)$ & $20(35)$ & $27(28)$ \\
$\quad 1-2$ & $17(44)$ & $21(37)$ & $16(28)$ \\
$\quad>2$ & $11(28)$ & &
\end{tabular}

Table 2. Dependence and barriers to quitting smoking

\begin{tabular}{|c|c|c|c|}
\hline Variable & Female & Male & Total \\
\hline Fagerström dependence score $(N=90)$, median (IQR) & $5(2-7)^{*}$ & $6(4-8)^{*}$ & $6(3-7)$ \\
\hline Exhaled CO (ppm), median (IQR) & $15(9-23)$ & $14(9-20)$ & $14.5(9-20)$ \\
\hline Motivation score (1 - 10), median (IQR) & $10(8-10)$ & $10(6-10)$ & $10(8-10)$ \\
\hline \multicolumn{4}{|l|}{ Reasons for quitting smoking $(N=98), n(\%)$} \\
\hline Personal health & $21(54)$ & $22(39)$ & $43(44)$ \\
\hline Financial reasons & $3(8)$ & $1(2)$ & $4(4)$ \\
\hline Family & $1(3)$ & $3(5)$ & $4(4)$ \\
\hline Fertility & 0 & $1(2)$ & $1(1)$ \\
\hline PHQ-9 depression score $(N=55)$, median (IQR) & $8(4-11)$ & $8(5-11)$ & $8(4-11)$ \\
\hline \multicolumn{4}{|l|}{ PHQ-9 total score $(N=55), n(\%)$} \\
\hline $0-4$ & $9(41)$ & $6(18)$ & $15(27)$ \\
\hline $5-9$ & $8(36)$ & $11(33)$ & $19(35)$ \\
\hline $10-15$ & $2(9)$ & $9(27)$ & $11(20)$ \\
\hline$>15$ & $3(14)$ & $7(21)$ & $10(18)$ \\
\hline \multicolumn{4}{|l|}{ Wisconsin Withdrawal Scale subscore, median (IQR) } \\
\hline Anger $(N=87)$ & $2.33(1.3-3)$ & $2(1.33-3.3)$ & $2(1.3-3)$ \\
\hline Anxiety $(N=90)$ & $2.5(1.8-3)$ & $2.25(1.3-2.8)$ & $2.25(1.25-3)$ \\
\hline Sadness $(N=89)$ & $2(1.3-2.6)$ & $1.75(1-2.5)$ & $2(1.25-2.5)$ \\
\hline Patient could potentially afford to purchase medication $(N=87), n(\%)$ & $10(28)$ & $18(35)$ & $28(32)$ \\
\hline
\end{tabular}

less strongly ( $r=0.41$ ( $95 \%$ CI 0.15 - 0.62); $p=0.02$ ). Current number of cigarettes per day correlated strongly with the Fagerström score $(r=0.74$ (95\% CI $0.62-0.82) ; p<0.001$ ) (Fig. 2). Motivation to quit was reported as high, and there was no difference in in that regard between men and women.

Depressive symptoms were prospectively evaluated, and complete scores were evaluable for 55 attendees at the first visit using the PHQ-9 depression test. Nearly two-thirds (63\%) of smokers reported at least minor symptoms of depression when screened at the first visit, and $38 \%(21 / 55)$ expressed symptoms of at least moderately severe depression (score $\geq 15$ ) (Fig. 3); 18\% (10/55) would be classified as severely depressed based on the PHQ-9 score. Evaluating the individual specified subcategories making up the Wisconsin
Withdrawal Scale at the initial visit (when the participants were still smoking), patients had on average high (>2/4) subscores for anger, anxiety and sadness (Table 2).

Smokers who had attended the clinic were contacted by telephone in 2016/17. Using the contact numbers provided at the first visit, only $46(47 \%)$ of the 97 attendees were contactable. The median time to follow-up was 22.5 months (IQR 14 - 39) after the first clinic visit. Based on self-reported smoking status, 13 attendees (28\%) had successfully quit, 33 (72\%) were still smoking, and 2 were stated to have died (cause not reported). Although the review was not specifically powered to evaluate predictors of outcome, individuals who had successfully quit had lower mean (SD) PHQ-9 depression scores than those who had been unsuccessful (3.9 (1.9) v. 7.1 (1.0), 


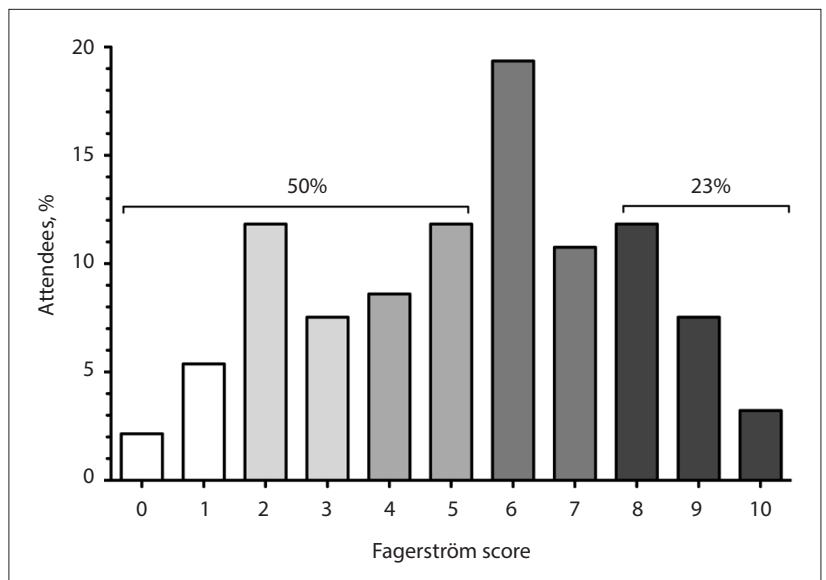

Fig. 1. Fagerström scores for nicotine dependence. (Interpretation of dependence scores: 0 - 1 low, 2 - 3 average, 4 - 5 above average, 6 - 7 high, 8 - 10 extreme nicotine dependence.)

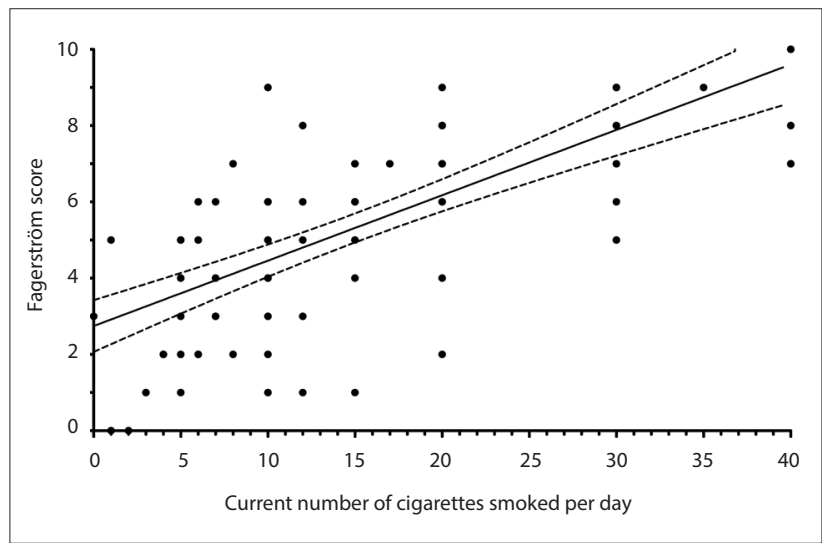

Fig. 2. Correlation between current daily number of cigarettes smoked and Fagerström score for nicotine dependence. Solid line: regression line with 95\% CI, Spearman's $\mathrm{r}=0.74$ (95\% CI $0.62-8.82)$; $\mathrm{p}<0.0001$. (CI = confidence interval.)

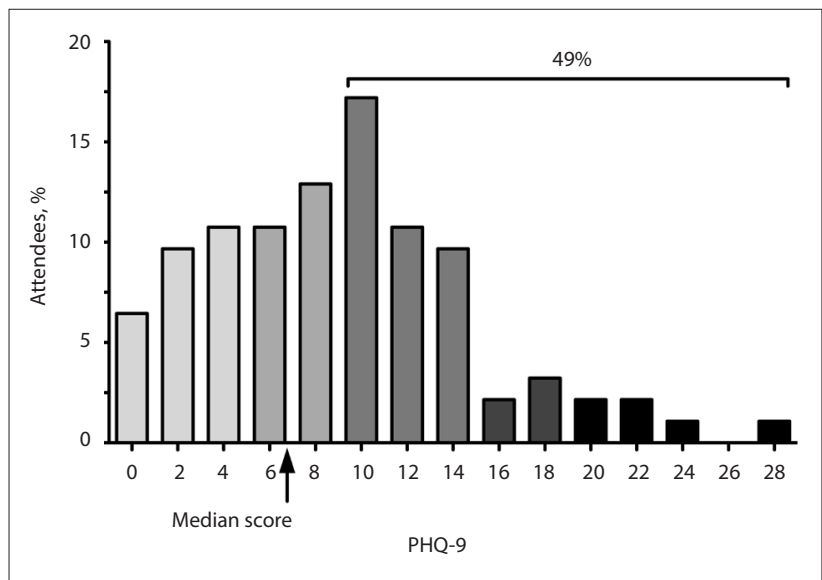

Fig. 3. PHQ-9 depression scores. (Interpretation of PHQ-9 score: 0 - 4 no symptoms, 5 - 9 minimal symptoms, 10 - 14 minor depression/major depression mild, 15 - 19 major depression moderately severe, $>20$ major depression severe; PHQ-9 = Patient Health Questionnnaire-9.)

respectively; $p=0.046$ ). In univariate analysis this translated into a trend towards successful quitting with a lower depression score (odds ratio $0.86(95 \% \mathrm{CI} 0.76-1.00) ; p=0.056)$. There was no difference in the number of clinic visits attended by those who quit successfully v. those who did not, or in duration of follow-up when contacted. Time to first cigarette in the morning was not predictive of success, and no difference was seen between quitters and non-quitters with regard to baseline nicotine dependence scores (Fagerström score 4 v. $5 ; p=0.34$ ) (Fig. 4, A and B).

\section{Discussion}

Access to formal smoking cessation services is very limited in LMICs. ${ }^{[16]}$ In this cohort of smokers from a low- to middleincome population group in SA attending a dedicated smoking cessation service, high levels of depressive symptoms were common, with accompanying moderate nicotine dependence. Currently no pharmacotherapy is provided by the government to assist smokers in quitting. Given the high level of depressive symptoms and stressors with relatively low nicotine dependence reported by the attendees, psychological and behaviour support for smokers should be a priority. The role for pharmacological support, given its poor availability and high cost, needs to be prospectively determined. Despite the lack of pharmacotherapy or specialised psychological support to assist in smoking cessation, some patients were successful in quitting smoking.

Global smoking cessation guidelines, including the SA guidelines, recommend a combination of behavioural support/counselling and pharmacotherapy to support smoking cessation attempts. ${ }^{[1,21,22]}$ The WHO has placed NRT on the essential medication list, but access to pharmacotherapy is severely limited and costly in resourceconstrained settings, even in a middle-income country such as SA.

To date there have been very limited data published on smoking cessation clinics in LMICs. The majority of reports are on integrated/ opportunistic brief interventions alongside healthcare services. ${ }^{[16,17]}$ Opportunities such as tuberculosis and HIV treatment programmes have shown promise as a chance to address tobacco smoking in conjunction with treating infectious diseases. ${ }^{[23,24]}$ In many developedworld settings, in-hospital smoking cessation strategies have been an opportunity to commence interventions. ${ }^{[25]}$ What is clear, however, is that if patients are not followed up or continued on treatment on discharge, all gains during the hospital stay with regard to quitting smoking are essentially for naught.

SA has been characterised as the world's second most stressful country to live in, although these data do not include much of Africa. ${ }^{[26]}$ If one compares the level of stressors in SA with other countries globally, however, Egypt (for example) is ranked 15th, Brazil 17th, China 29th, the UK 56th and Australia 70th. During counselling of the smokers in our study, common reported stressors included personal safety, financial insecurity, health concerns, community safety and anxieties regarding the country's future. These concerns may be intensified by a hospitalisation or acute medical illness, especially if an additional financial strain and burden is precipitated by lack of income and family support. ${ }^{[27]}$ Unexpected hospital admissions may therefore not be the best opportunities to intervene, given the accompanying heightened stress.

Smokers in our outpatient cohort showed significant levels of background depressive symptoms, both on the formal PHQ-9 depression scoring and also when we evaluated the subscore of the Wisconsin Withdrawal Scale (while the attendees were still smoking). In active smokers, a balance exists between tobacco/nicotine dependence and the psychological/ behavioural habit of smoking (although these aspects are not always clearly separable). There is strong evidence that counselling and pharmacotherapy go hand in hand, with a combination generally better than either alone. ${ }^{[21]}$ What 


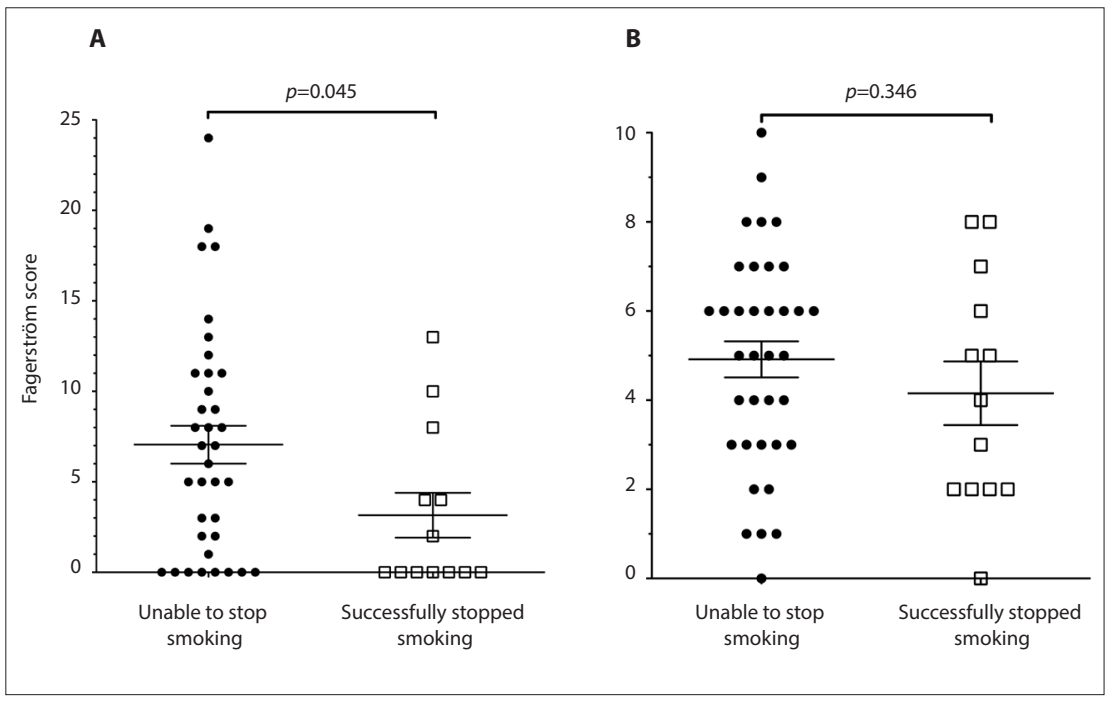

Fig. 4. Characteristics of patients with known outcomes at 2-year median follow up: (A) PHQ-9 depression score by outcome; (B) Fagerström nicotine dependence score by outcome (mean (SD)). (PHQ-9 = Patient Health Questionnnaire-9; SD = standard deviation.)

is not clear, and has no literature to support it, is how to estimate whether an individual will be able to quit without the addition of medication. There is a strong correlation between Fagerström score and long-term cessation success, ${ }^{[28]}$ and specifically time to first cigarette in the morning has been shown to be a simple measure to use. ${ }^{[28]}$ Additionally, underlying depression and poor outcomes are correlated in reports from smoking cessation services. ${ }^{[29]}$ In our cohort, no significant correlation was found between dependence indices and outcome, although the audit was not primarily powered to evaluate predictors of outcome owing to the nature of the study. Despite the lack of pharmacotherapy long-term quit rates were encouraging, although an estimation of true success was not possible owing to the excessive loss to follow-up and inability to confirm smoking status chemically. What was evident in this cohort of low-income smokers was the low to moderate level of nicotine dependence and moderate to high level of depressive symptoms. This finding raises the question whether it is preferable to spend resources on skilled counselling when establishing dedicated smoking cessation centres in lowincome settings, or on the provision of NRT/ varenicline/bupropion to aid in quit attempts for all patients.

\section{Study limitations}

The study has several limitations. Referral to the service was encouraged for smokers motivated to quit. The service did not have on-site specialised counselling for depression or anxiety, but relied on referral to such services. The level of depressive symptoms may be different in individuals who are not willing to quit, but the characteristics of the attendees presented were of those wanting help to stop smoking. It was not possible to evaluate whether outcomes would have been different if the patients had had access to pharmacotherapy. There is therefore uncertainty whether to prioritise counselling or medication, but the high level of depressive symptoms requires attention irrespective of smoking status. Follow-up was incomplete, as many of the contact telephone numbers that were provided did not work. Prespecified powered outcome calculations were not possible, but patient data collection is ongoing.

\section{Recommendations}

The questions facing the clinician with limited access to pharmacotherapy to assist smoking cessation are who should receive it, and whether it is possible to predict who will not be successful without the provision of medication. An approach could be to initiate an intensive behaviour motivation intervention irrespective of dependence scores, and to provide NRT at the second attempt for those who fail due to withdrawal symptoms. The strategy in our clinic was to provide a support service that did not previously exist in an attempt to help smokers quit, and to gather the necessary data to motivate for locally relevant psychological and/or pharmacological interventions. Based on the data from this cohort, attending to the social, financial and emotional upheaval described by the majority of smokers seen may be of far more importance than pharmacotherapy in achieving long-term freedom from tobacco dependence. Poverty alleviation, sustainable income and mental health are not issues that can simply be solved by a nicotine patch or gum. Providing behavioural tools to assist with coping with life stressors, counselling and appropriate referral to social services is a starting point to explore in low-income settings where the behavioural aspects of tobacco dependence as a coping mechanism may outweigh the pure chemical addiction.

As with all interventions in medicine, the certainty with which outcomes/response to therapy can be predicted enhances our decision-making and ability to motivate to funders to support treatment strategies. In low-resourced areas, the clinician is bound to equitable resource allocation and often provides interventions for those who will 'benefit' the most. If we could reasonably identify those who will inevitably fail without pharmacotherapy, we could motivate for such targeted interventions. Extrapolating from the nicotine dependence scores in this cohort, $\sim 25 \%$ had very high dependence and should by any major guideline strategy have been receiving pharmacological support. The PHQ-9 scores indicative of major depression in $\sim 1$ in 5 smokers would warrant appropriate referral to competent psychiatric services before any quit attempt is made. Depending on the skills of the service providers (doctor v. psychologist), these services could be interlinked.

\section{Conclusions}

Smoking cessation should be a priority in all healthcare services, especially in lowincome settings where smoking results in a significant health burden, drives mortality rates and consumes substantial disposable income among those who can least afford it. High levels of depression and social stressors existed in our cohort of smokers, suggesting that interventions targeting the behavioural aspects of tobacco dependence should be emphasised and potentially balanced with the provision of NRT for those with very high dependence. Prospective, scalable and cost-effective interventions for tobacco dependence are needed to address this major modifiable risk factor for communicable and non-communicable diseases in low-income settings.

\section{Declaration. None.}

Acknowledgements. We thank the E16 staff who assisted with establishing the clinic.

Author contributions. RNvZS established the audit, AE, CD, RNvZS and CC collected the 
data, MK, RNvZS and GYT analysed the data, and all authors reviewed the manuscript prior to publication.

Funding. GYT was supported by a South African Thoracic SocietyNovartis training fellowship. RNvZS received funding from the Cape Higher Education Consortium - City of Cape Town research programme. Conflicts of interest. RNvZS received honoraria from Pfizer for smoking cessation-related academic presentations.

1. Doll R, Hill AB. Smoking and carcinoma of the lung. BMJ 1950;2(4682):739-748. https://doi.org/10.1136/ bmj.2.4682.739

2. Lee PN, Forey BA, Coombs KJ. Systematic review with meta-analysis of the epidemiological evidence in the 1900s relating smoking to lung cancer. BMC Cancer 2012;12:385. https://doi.org/10.1186/1471 2407-12-385

3. Forey BA, Thornton AJ, Lee PN. Systematic review with meta-analysis of the epidemiological evidence relating smoking to COPD, chronic bronchitis and emphysema. BMC Pulm Med 2011;11:36. https://doi org/10.1186/1471-2466-11-36

4. Marufu TC, Ahankari A, Coleman T, Lewis S. Maternal smoking and the risk of still birth: Systematic review and meta-analysis. BMC Public Health 2015;15:239. https://doi.org/10.1186/s12889-015-1552-5 5. Fretts RC. Etiology and prevention of stillbirth. Am J Obstet Gynecol 2005;193(6):1923-1935. https://doi. org/10.1016/j.jog.2005.03.074

6. Cnattingius S. The epidemiology of smoking during pregnancy: Smoking prevalence, maternal characteristics, and pregnancy outcomes. Nicotine Tob Res 2004;6(Suppl 2):S125-S140. https://doi.org characteristics, and pregnancy outc

7. Bradshaw D, Groenewald P, Laubscher R, et al. Initial burden of disease estimates for South Africa, 2000. 7. Bradshaw D, Groenewald P, Laub

8. Groenewald P, Vos T, Norman R, et al. Estimating the burden of disease attributable to smoking in South 8. Groenewald P, Vos T, Norman R, et al. Estimating
Africa in 2000. S Afr Med J 2007;97(8):674-681.

Africa in 2000. S Afr Med J 2007;97(8):674-681.
9. Joint United Nations Programme on HIV and AIDS (UNAIDS). Aids info. http://aidsinfo.unaids.or 9. Joint United Nations Pro
(accessed 23 April 2019).

10. Wang J, Shen H. Review of cigarette smoking and tuberculosis in China: Intervention is needed for smoking cessation among tuberculosis patients. BMC Public Health 2009;9:292. https://do org/10.1186/1471-2458-9-292

11. Kwan CK, Ernst JD. HIV and tuberculosis: A deadly human syndemic. Clin Microbiol Rev 2011;24(2):351-376. https://doi.org/10.1128/CMR.00042-10

12. Bates MN, Khalakdina A, Pai M, Chang L, Lessa F, Smith KR. Risk of tuberculosis from exposure to tobacco smoke: A systematic review and meta-analysis. Arch Intern Med 2007;167(4):335-342. https:// doi.org/10.1001/archinte.167.4.335

13. Van Zyl Smit RN, Pai M, Yew WW, et al. Globallung health: The colliding epidemics of tuberculosis, tobacco Van Zyl Smit RN, Pai M, Yew WW, et al. Global lunghealth: The colliding epidemics of tuberculosis, tobace
smoking, HIV and COPD. Eur Respir J 2010;35(1):27-33. https://doi.org/10.1183/09031936.00072909
14. Banks E, Joshy G, Weber MF, et al. Tobacco smoking and all-cause mortality in a large Australian cohort study: Findings from a mature epidemic with current low smoking prevalence. BMC Med 2015;13:38. sudy: Find go ro

5. World Health Organization. WHO Report on the Global Tobacco Epidemic, 2015. Geneva: WHO, 2015. https://www.who.int/tobacco/global_report/2015/en/ (accessed 18 April 2019).

16. Ward KD. Tobacco intervention research in low- and middle-income countries: Lessons learned and future directions. J Smok Cessat 2016;11(2):61-64. https://doi.org/10.1017/jsc.2016.6

17. Lando H. Promoting tobacco cessation in low- and middle-income countries. J Smok Cessat 2016;11(2):66-69. https://doi.org/10.1017/jsc.2016.7

18. World Health Organization. Tobacco atlas online 2019. http://www.tobaccoatlas.org (accessed 18 April 2019).

19. Van Zyl-Smit RN, Allwood B, Stickells D, et al. South African tobacco smoking cessation clinical practice guideline. S Afr Med J 2013;103(11):869-876. https://doi.org/10.7196/SAMJ.7484

20. Heatherton TF, Kozlowski LT, Frecker RC, Fagerström KO. The Fagerström Test for Nicotine Dependence: A revision of the Fagerström Tolerance Questionnaire. Br I Addict 1991;86(9):1119-1127. https://doi.org/10.1111/j.1360-0443.1991.tb01879.x

21. Fiore MC. Treating tobacco use and dependence: 2008 update US Public Health Service Clinical Practice Guideline executive summary. Respir Care 2008;53(9):1217-1222.

22. Hughes JR. An updated algorithm for choosing among smoking cessation treatments. J Subst Abuse Treat Hughes JR. An updated algorithm for choosing among smoking

23. Khan AH, Israr M, Khan A, Aftab RA, Khan TM. Smoking on treatment outcomes among tuberculosis Khan AH, Israr M, Khan A, Aftab RA, Khan TM. Smoking on treatment outcomes among tut
patients. Am J Med Sci 2015;349(6):505-509. https://doi.org/10.1097/MAJ.0000000000000473

24. Nahvi S, Cooperman NA. Review: The need for smoking cessation among HIV-positive smokers. AIDS Educ Prev 2009;21(3 Suppl):14-27. https://doi.org/10.1521/aeap.2009.21.3_supp.14

25. Rigotti NA, Clair C, Munafo MR, Stead LF. Interventions for smoking cessation in hospitalised patients. Cochrane Database Syst Rev 2012, Issue 5. Art. No.: CD001837. https://doi.org/10.1002/14651858. CD001837.pub3

6. Bloomberg. Most stressed out countries 2013. http://www.bloomberg.com/graphics/best-andworst/\#most-stressed-out-countries (accessed 19 April 2019).

27. Tanimura T, Jaramillo E, Weil D, Raviglione M, Lonnroth K. Financial burden for tuberculosis patients in low- and middle-income countries: A systematic review. Eur Respir J 2014;43(6):1763-1775. https:// doi.org/10.1183/09031936.00193413

28. Baker TB, Piper ME, McCarthy DE, et al.; Transdisciplinary Tobacco Use Research Center (TTURC) Tobacco Dependence Phenotype Workgroup. Time to first cigarette in the morning as an index of ability tobacco Dependence Phenotype Workgroup. Time to first cigarette in the morning as an index of ability
to quit smoking: Implications for nicotine dependence. Nicotine Tob Res 2007;9(Suppl 4):S555-S570, to quit smoking: Implications for nicotine

29. Sonne SC, Nunes EV, Jiang H, Tyson C, Rotrosen J, Reid MS. The relationship between depression and smoking cessation outcomes in treatment-seeking substance abusers. Am J Addict 2010;19(2):111-118. https://doi.org/10.1111/j.1521-0391.2009.00015.x

Accepted 12 December 2018 